\title{
Synchronous photoinjection using a frequency-doubled gain-switched fiber-coupled seed laser and ErYb-doped fiber amplifier
}

\author{
J. Hansknecht* and M. Poelker \\ Thomas Jefferson National Accelerator Facility, 12000 Jefferson Avenue, Newport News, Virginia 23606, USA
}

(Received 12 April 2006; published 21 June 2006)

\begin{abstract}
Light at $1560 \mathrm{~nm}$ from a gain-switched fiber-coupled diode laser and ErYb-doped fiber amplifier was frequency doubled to obtain over $2 \mathrm{~W}$ average power at $780 \mathrm{~nm}$ with $\sim 40 \mathrm{ps}$ pulses and pulse repetition rate of $499 \mathrm{MHz}$. This light was used to drive the $100 \mathrm{kV}$ DC high voltage GaAs photoemission gun at the Continuous Electron Beam Accelerator Facility at Jefferson Laboratory to produce a high average current beam $(100 \mu \mathrm{A})$ of highly spin-polarized electrons $(>80 \%)$. This new drive-laser system represents a significant advance over laser systems used previously, providing significantly higher power and enhanced reliability.
\end{abstract}

DOI: 10.1103/PhysRevSTAB.9.063501

PACS numbers: 29.27.Hj, 42.55.Wd, 29.25.Bx

\section{INTRODUCTION}

The Continuous Electron Beam Accelerator Facility (CEBAF) at the Thomas Jefferson National Accelerator Facility (Jefferson Lab) is a unique electron accelerator for nuclear physics research, providing experimenters the highest current polarized electron beam available worldwide. Beamtime is oversubscribed and the physics program cannot accommodate downtime associated with maintenance of the $100 \mathrm{kV}$ DC high voltage GaAs photogun that provides beam for all experiments. As such, prolonging photocathode lifetime has always been a central concern. Synchronous photoinjection is one strategy employed at CEBAF to prolong photocathode lifetime. The photogun drive lasers emit picosecond optical pulses synchronized to the accelerator cavity rf. Nearly all of the extracted electrons are delivered to the experimental halls, very few electrons are extracted and then thrown away as happens when using DC light. Thus photocathode $Q E$ degrades more slowly because the average beam current is kept to a minimum. Synchronous photoinjection with a DC high voltage GaAs photogun was first demonstrated during 1996 at CEBAF [1]. Since then, it has been implemented at the Mainz Microtron [2] and the Jefferson Lab FEL [3]. Many proposed accelerator facilities expect to employ synchronous photoinjection [4-7].

CEBAF uses superconducting accelerating cavities at $1497 \mathrm{MHz}$. Each experimental hall receives an electron pulse train at $499 \mathrm{MHz}$, the third subharmonic of the fundamental accelerating frequency. Implementing synchronous photoinjection at CEBAF therefore requires drive lasers with very high pulse repetition rates that were not commercially available during the mid-1990s when the polarized photoinjector at CEBAF was being constructed. The first rf-pulsed laser system developed at Jefferson lab was a diode laser master oscillator power amplifier

\footnotetext{
*Corresponding author.

Electronic address: hansknec@jlab.org
}

(MOPA) composed of a gain-switched diode seed laser and single pass tapered-stripe diode amplifier [8]. The diode MOPA system was very reliable, providing light at $780 \mathrm{~nm}$ or $840 \mathrm{~nm}$, with $50 \mathrm{ps}$ pulses and pulse repetition rates at $499 \mathrm{MHz}$ or $1497 \mathrm{MHz}$. The optical pulse train was easily phase locked to the accelerator but output power was limited to $50 \mathrm{~mW}$, not enough to provide the required $100 \mu \mathrm{A}$ of highly polarized beam from high polarization photocathode material. To address the power limitation of the diode MOPA system, a novel harmonic-modelocked Ti-sapphire laser was invented [9]. This laser satisfied the optical power requirements and could be wavelength tuned to provide maximum beam polarization, but the output beam was noisy which introduced undesirable highfrequency amplitude modulation on the extracted electron beam. The exact cause of the laser power instability was never fully understood but was likely related to the absence of active laser cavity length stabilization, which was not developed because a commercial modelocked Ti-sapphire laser with $499 \mathrm{MHz}$ pulse repetition rate became available from TimeBandwidth Products [10]. This commercial laser system relies on passive modelocking using a semiconductor saturable absorber mirror (SESAM) and was purchased with cavity length stabilization electronics that locks the optical pulse train to an external reference frequency (i.e., the accelerator) with integrated phase noise $<1$ ps. Laser cavity elements can be swapped to produce tunable light at wavelengths centered near 780 and $850 \mathrm{~nm}$ with output power $\sim 500 \mathrm{~mW}$. Unfortunately the commercial modelocked Ti-sapphire laser requires maintenance by lab staff typically once per week, to realign the laser cavity mirrors to minimize oscillation at higher order spatial modes and/or to obtain oscillation at new locations on the SESAM that sometimes gets damaged via inadvertent $Q$-switching.

The fiber-based laser system described in this paper represents a very significant contribution to the field of drive-laser technology for polarized GaAs photoguns. This laser system offers numerous advantages compared to the 
low power diode MOPA system and modelocked Tisapphire lasers described above. In particular, the fiberbased laser system provides considerably more power than modelocked Ti-sapphire lasers but requires very little maintenance. It relies on the accelerator-friendly technique of gain switching (described below) to create the high repetition rate optical pulse train that is easily phase locked to the accelerator rf cavities, without complicated feedback loops. Gain switching provides ideal laser parameters for many accelerator applications; $\sim 40$ ps pulses at repetition rates between 0.1 to $3 \mathrm{GHz}$ without modification to the setup. Many of the laser components are readily available and relatively inexpensive because the telecom industry spurs development of fiber components at $1560 \mathrm{~nm}$ wavelength. The fiber amplifier produces very little amplified spontaneous emission (ASE) when seeded with light at rf repetition rates compatible with gain switching. Moreover, the small amount of ASE that leaves the fiber amplifier is not efficiently frequency doubled to $780 \mathrm{~nm}$, ensuring that very little DC beam is generated at the photocathode. The use of the fiber-based laser system at $1560 \mathrm{~nm}$ is a logical step that comes hand-in-hand with the recent development of high polarization superlattice GaAs photocathode material requiring light near $780 \mathrm{~nm}$ [11].

\section{SYSTEM DESCRIPTION AND RESULTS}

The fiber-based laser system consists of three main components; the gain-switched fiber-coupled diode seed laser, the ErYb-doped fiber amplifier, and the periodically poled lithium niobate (PPLN) frequency doubler (Fig. 1). Fiber-coupled seed lasers at wavelength $1560 \mathrm{~nm}$ are inexpensive components, widely used within the telecom industry. The seed laser used in this experiment was a single-longitudinal mode distributed feedback Bragg reflector laser (DFB), rated for $20 \mathrm{~mW}$ average output power at normal DC operating current, mounted within a "butterfly" package with fiber-coupled output and internal optical isolator to prevent damage due to retroreflected light. The butterfly package provides an internal photodiode for noninvasive power monitoring and a thermoelectric cooler for temperature stabilization and wavelength tuning. The fiber pigtail, as it is known, is a single-mode polarizationmaintaining fiber with an FC connector [12] to mate with the input connector of the ErYb-doped fiber amplifier described below.

Gain switching is a purely electrical technique [13] where the diode laser is biased near threshold and driven with rf energy through a bias network. The gain-switched diode laser produces pulses at a repetition rate equal to the rf drive frequency. Optical pulse width is determined mostly by the laser cavity length (and photon lifetime), a parameter set by the vendor; 30-100 ps pulses are common. Our experience to date indicates that the best optical pulses (i.e., narrow, without secondary pulses) are obtained when the rf sine wave signal is passed through a comb

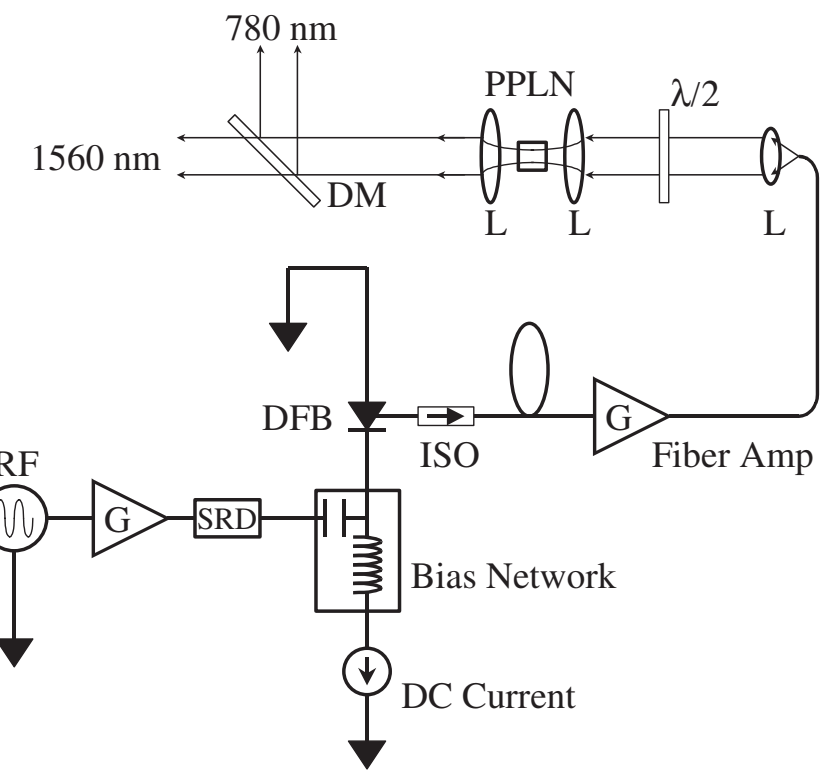

FIG. 1. Schematic of the fiber-based laser system. DFB, distributed feedback Bragg reflector diode laser; ISO, fiber isolator; SRD, step recovery diode; L, lens; PPLN, periodicallypoled lithium niobate frequency-doubling crystal; DM, dichroic mirror.

generator (also known as step recovery diode, SRD), to drive the diode laser with a train of short electrical pulses [14]. The pulse repetition rate can be varied over a wide range of frequencies from 0.1 to $3 \mathrm{GHz}$, although slight adjustment of the DC bias level and rf amplitude is required to maintain optimum pulse shape. In addition, the SRD must function properly at the desired operating frequency. The seed laser produces $\sim 1 \mathrm{~mW}$ average power at $499 \mathrm{MHz}$, a value that scales with duty factor.

The fiber pigtail of the seed laser was attached to the mated input connector of the commercial fiber amplifier [15] which consists of three cascaded ErYb-fiber amplifiers, each pumped with light from high power diode lasers. The amplifier is a "turn-key" device, without user serviceable components that provides up to $38 \mathrm{dBm}$ gain and maximum output power of $6 \mathrm{~W}$ at $1560 \mathrm{~nm}$ for input power within the range of 0.1 to $1 \mathrm{~mW}$ ( -10 to $0 \mathrm{dBm}$ ) between 1545 and $1565 \mathrm{~nm}$. The amount of amplification can be set by adjusting the current to the pump laser of the final stage of the amplifier. The amplifier input and output fibers are standard single-mode polarization-preserving with numerical aperture 0.11, terminated with FC/APC [12] connectors. An FC fiber collimating lens with $11 \mathrm{~mm}$ focal length produces an output beam with $\sim 2 \mathrm{~mm}$ diameter (FWHM).

The beam from the fiber amplifier was directed into a temperature tuned periodically poled lithium niobate (PPLN) crystal $10 \mathrm{~mm}$ long [16]. A lens with $30 \mathrm{~mm}$ focal length provided a calculated beamwaist of $\sim 28 \mu \mathrm{m}$ at the center of the PPLN crystal and a Rayleigh range of $\sim 1.6 \mathrm{~mm}$, a value inconsistent with the rule-of-thumb 
recommendation of the PPLN vendor stating that optimum conversion efficiency is obtained when the crystal length is 2 times the Rayleigh range, but this focusing lens was inhouse and easy to mount near the PPLN oven. Efficient frequency doubling required adjustment of the trajectory of the $1560 \mathrm{~nm}$ light through the PPLN crystal, proper orientation of the input linear polarization using the half-wave plate upstream of the PPLN crystal, and temperature tuning of the PPLN crystal to obtain optimum phase matching for the two light beams at wavelengths 1560 and $780 \mathrm{~nm}$. The light that leaves the PPLN crystal was collimated using another $30 \mathrm{~mm}$ focal length lens. A dichroic mirror reflects the useful $780 \mathrm{~nm}$ light and passes residual $1560 \mathrm{~nm}$ light which is dumped. The performance of the system is shown in Fig. 2, where output power at $780 \mathrm{~nm}$ is plotted versus PPLN input power at $1560 \mathrm{~nm}$ for three input conditions; DC light and rf-pulsed light at 499 and $1497 \mathrm{MHz}$. The nonlinear frequency-doubling process favors high peak power, which of the three conditions tested corresponds to $499 \mathrm{MHz}$. The maximum conversion efficiency was $\sim 40 \%\left(P_{\text {out }} / P_{\text {in }}\right)$. Initially there was concern that the spectral bandwidth of the gain-switched seed light $(\sim 2 \mathrm{~nm}$ ) would be too wide to obtain high conversion efficiency [17] but $2 \mathrm{~W}$ average output power at $780 \mathrm{~nm}$ and $499 \mathrm{MHz}$ clearly exceeded our design goal of $500 \mathrm{~mW}$, the output power of the modelocked Ti-sapphire drive lasers we intend to replace.

Optical pulses were monitored at each stage of the laser system using a fast photodiode and oscilloscope with $\sim 100$ ps resolution bandwidth. The gain-switched seed laser produces detector-limited pulses (Fig. 3), similar pulses were observed downstream of the fiber amplifier and the PPLN frequency-doubler crystal. The detector system cannot resolve the narrowing of pulse width that likely occurs during the frequency-doubling process. More

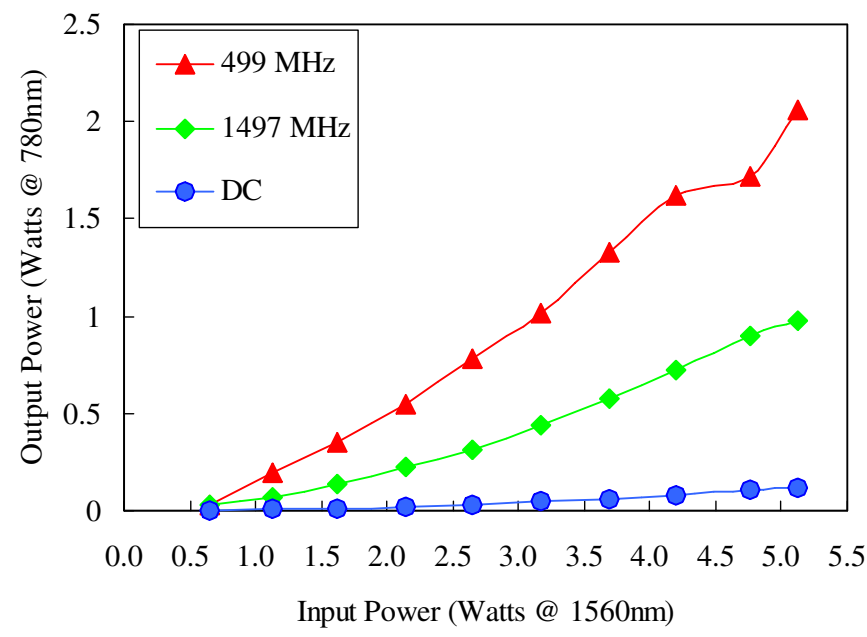

FIG. 2. (Color) Output power of the fiber-based laser system at $780 \mathrm{~nm}$ versus input power from the seeded ErYb-doped fiber amplifier at $1560 \mathrm{~nm}$. Three different seed conditions were tested; DC and rf-pulsed input at 499 and $1497 \mathrm{MHz}$.

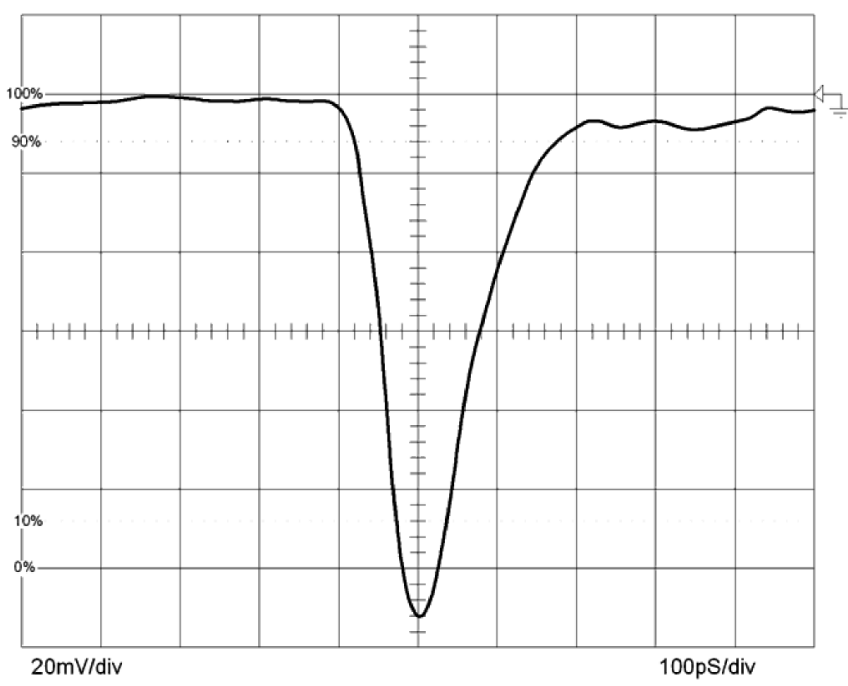

FIG. 3. Detector-limited photodiode signal of gain-switched seed laser at $1560 \mathrm{~nm}$ and $499 \mathrm{MHz}$ pulse repetition rate. Similar photodiode signals were observed downstream of the fiber amplifier and PPLN frequency-doubler crystal; actual pulse width is narrower, as described in text.

accurate pulse width monitoring was performed at the CEBAF photoinjector described below.

\section{OPERATION AT CEBAF}

The fiber-based laser system was installed at the CEBAF photoinjector, with $780 \mathrm{~nm}$ light directed toward the GaAs superlattice photocathode within the $100 \mathrm{kV}$ DC high voltage photogun. The light passes through a pockels cell that converts linearly polarized light to circularly polarized light, a necessary condition for generating spin-polarized electrons. The fiber amplifier produces light with very good spatial beam quality (nearly diffraction limited), which is maintained following frequency doubling. The $30 \mathrm{~mm}$ focal length lens at the output of the PPLN crystal was used to adjust the collimation of the laser beam. A $2 \mathrm{~m}$ focal length lens near the photogun vacuum chamber window creates a $500 \mu \mathrm{m}$ diameter (FWHM) focused spot at the photocathode.

The photogun produced electron bunches that were monitored using the CEBAF photoinjector chopping system, which consists of two $499 \mathrm{MHz} \mathrm{TM}_{210}$ mode rf deflecting cavities and a sophisticated UHV-compatible 3 -slit apparatus located midway between the two rf cavities that sets the maximum phase acceptance of the accelerator. The first rf deflecting cavity sweeps the electron beam in a circle across the slit apparatus, a water-cooled copper plate with three apertures spaced $120^{\circ}$ apart. The second $\mathrm{rf}$ deflecting cavity restores the initial beam trajectory. Under normal three-hall operation, three drive lasers produce interleaved $499 \mathrm{MHz}$ electron pulse trains. The phase of each drive laser is set to pass the corresponding electron beam through one of the slits. For the electron bunchlength 
measurements reported here, two of the slits were closed and the other was set to a very narrow opening, approximately $3^{\circ}$ of rf phase at $499 \mathrm{MHz}$, enough to pass beam within a temporal window $11 \mathrm{ps}$ wide. The rf phase of the fiber-based laser system was varied to move the electron bunch across the narrow slit, with the transmitted beam delivered to a Faraday cup connected to a picoammeter. Laser-phase scans at four beam currents are shown in Fig. 4(a), with the horizontal phase axis converted to units of time. Coulomb repulsion causes the electron bunchlength to grow as a function of beam current, behavior noted early in the polarized beam program at CEBAF using other drive lasers [1]. At low current, where the coulomb repulsion force is small, the electron bunchlength mimics the optical pulse shape. Figure 4(b) shows the $1 \mu \mathrm{A}$ data with a Gaussian fit. The electron bunch length, and similarly the optical pulse width at $780 \mathrm{~nm}$, is 37 ps (FWHM) when the width of the narrow chopper slit is deconvolved from the measurement. The pulse width at $1560 \mathrm{~nm}$ must be $53 \mathrm{ps}$, assuming a reduction in pulse width by $\sqrt{2}$
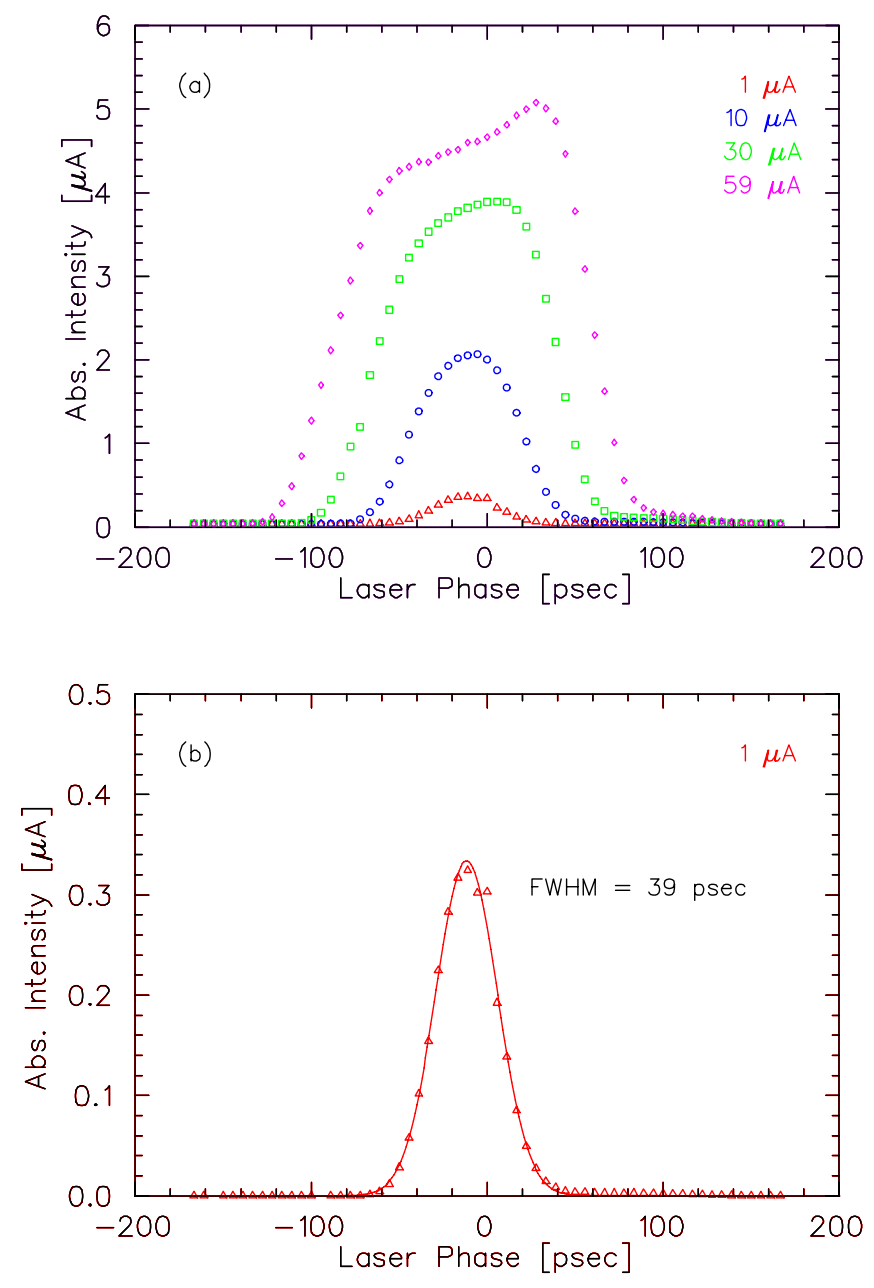

FIG. 4. (Color) (a) Electron bunchlength measurements at four different beam currents and (b) the $1 \mu \mathrm{A}$ data with Gaussian fit. associated with the frequency-doubling process and Gaussian pulse.

The chopper system was also used to detect the presence of DC light produced by the fiber-based laser system. DC light is undesirable because it produces electrons that are merely thrown away, hastening photocathode $Q E$ decay. In addition, DC light is problematic at CEBAF where three halls can receive beam simultaneously. The complete drive-laser system is composed of three independent lasers (one for each experimental hall) and beam-combining optics that place each laser beam on a common path directed toward the same location on the photocathode. The light from each drive laser does not necessarily have the same polarization. Efficient laser beam combining sometimes requires using drive lasers with orthogonal beam polarization or dissimilar wavelengths. As a result, the DC beam created by one laser can have opposite or greatly reduced beam polarization compared to that created by another drive laser. This leads to dilution of beam polarization at individual halls, a condition most evident at halls requesting low beam current. To quantify the amount of DC beam created by the new laser, the rf phase of the fiber-based laser system was set to dump the electron bunches at a closed chopper slit, while current through fully open adjacent slits (combined acceptance $40^{\circ}$ of $\mathrm{rf}$ phase at $499 \mathrm{MHz}$ ) was monitored using the Faraday cup and picoammeter. The current that passes through the open slits must originate from DC light and was measured to be $\sim 4 \mathrm{nA}$ compared to $50 \mu \mathrm{A}$ dumped at the closed chopper slit. Extrapolating to $360^{\circ}$ of rf phase and assuming that the optical pulses from the fiber-based laser system ride atop a continuous low level of DC yields $36 \mathrm{nA}$ of DC beam, more than 1000 times less than the useful rf-pulsed beam delivered to the accelerator. This level of DC beam is acceptable for CEBAF; significantly smaller than DC light produced by the diode MOPA systems and comparable to the amount of DC beam produced by the modelocked Tisapphire lasers.

\section{CONCLUSION}

Shortly after initial tests, the fiber-based laser system was used for production beam delivery to two halls. Both halls report high beam polarization $>80 \%$ and good beam current stability. The fiber-based laser has been in constant use for eight weeks, operating reliably without interruption or maintenance, with the optical pulse train precisely phase locked to the accelerator rf without feedback. There is every indication that this laser system is perfectly suited for CEBAF, providing more power and enhanced reliability compared to diode MOPA systems and modelocked Tisapphire lasers used previously.

Other accelerators will likely benefit from this laser. Gain switching is dramatically more reliable than modelocking and can be implemented at L-band and S-band frequencies. The fiber-based laser system provides 4 times 
as much power as today's modelocked Ti-sapphire laser systems and is perhaps the highest average power drive laser ever constructed for polarized beam delivery. Continued advances in fiber amplifier technology will likely push maximum available output power considerably beyond $2 \mathrm{~W}$ reported here. In addition, readily available telecom components may provide a means to inexpensively shape electron bunches for emittance compensation for high bunch charge applications of future accelerator designs. Finally, it is reasonable to assume that a similar system with gain-switched seed laser can be built at $1.06 \mu \mathrm{m}$, for frequency doubling to $532 \mathrm{~nm}$ for bulk GaAs and unpolarized beam applications [18].

\section{ACKNOWLEDGMENTS}

The authors are grateful for helpful discussions and advice from Dr. Glen Williams of Keopsys Inc., and Dr. Lew Goldberg of the Naval Research Lab. This work was supported by the U.S. DOE under Contract No. DEAC05-84ER401050.

[1] M. Poelker, in Proceedings of the 12th International Symposium on High-Energy Spin Physics (SPIN 96), Amsterdam, Netherlands, 1996, p. 723; M. Poelker and J. Hansknecht, Proceedings of the Workshop on Polarized Gas Targets and Polarized Beams [AIP Conf. Proc. 421, 270 (1997)].

[2] K. Aulenbacher, H. Euteneuer, D. v. Harrach, P. Hartmann, J. Hoffmann, P. Jennewein, K. H. Kaiser, H. J. Kreidel, H. J. Leberig, C. Nachtigall, E. Reichert, M. Schemies, J. Schuler, M. Steigerwald, and C. Zalto, in Proceedings of the 6th European Particle Accelerator Conference (EPAC98), edited by S. Meyers, L. Lilijeby, C. PetitJean-Genaz, J. Poole, and K.-G. Rensfeldt (Institute of Physics Publishing, Bristol and Philadelphia, 1998), pp. 1388-1390.

[3] S. Benson, G. Biallas, C. Bohn, D. Douglas, H. F. Dylla, R. Evans, J. Fugitt, R. Hill, K. Jordan, G. Krafft, R. Legg, R. Li, L. Merminga, G. R. Neil, D. Oepts, P. Piot, J. Preble, M. Shinn, T. Siggins, R. Walker, and B. Yunn, Nucl. Instrum. Methods Phys. Res., Sect. A 429, 27 (1999); other JLab FEL-related publications are available at http://www.jlab.org/FEL/felpubs/.

[4] I. Bazarov and C. K. Sinclair, in Proceedings of the 2003 IEEE Particle Accelerator Conference (PAC2003),
Portland, Oregon (2003), p. 2062.

[5] C. Gerth and F. E. Hannon, in Proceedings of the 2004 IEEE European Particle Accelerator Conference (EPAC2004), Lucerne, Switzerland (2004), p. 437.

[6] I. Ben-Zvi, Ya. Derbenev, V. N. Litvinenko, and L. Merminga, in Proceedings of the 32nd Advanced ICFA Beam Dynamics Workshop on Energy Recovering Linacs, Newport News, VA (2005), p. 28 [Nucl. Instrum. Meth. A 557, 28 (2006)].

[7] L. Merminga and Ya. Derbenev, Conference on the Intersections of Particle and Nuclear Physics [AIP Conf. Proc. 698, 811 (2004)].

[8] M. Poelker, Appl. Phys. Lett. 67, 2762 (1995).

[9] C. Hovater and M. Poelker, Nucl. Instrum. Methods Phys. Res., Sect. A 418, 280 (1998), U.S. Patent No. 6483858.

[10] Time-Bandwidth Products, Inc., Halle Zeppelin 1. OG, Technoparkstrasse 1, CH-8005 Zurich, Switzerland, http:// www.timebandwidth.com.

[11] T. Maruyama, D.-A Luh, A. Brachmann, J.E. Clendenin, E. L. Garwin, S. Harvey, J. Jiang, R. E. Kirby, C. Y. Prescott, R. Prepost, and A. M. Moy, Appl. Phys. Lett. 85, 2640 (2004); J.E. Clendenin, in Proceedings to the 10th Workshop on Polarized Sources and Targets, Novosibirsk, Russia (2003) [Nucl. Instrum. Methods Phys. Res., Sect. A 536, 308 (2005)].

[12] There are many types of fiber optic connectors including SMA, SC, ST, and FC. The FC connector is common, with keyed and threaded ferrule to preserve the orientation of the polarization axis of polarization-maintaining singlemode fibers. Other fiber optic designations include APC for angle physical contact, PM for polarization maintaining, and SM for single mode.

[13] See, for example, P. T. Ho, in Picosecond Optoelectronic Devices, edited by C.H. Lee (Academic, New York, 1984).

[14] This is contrary to our experience with free-space diode lasers where the SRD is not required; narrow optical pulses can be obtained by merely applying the rf sine wave directly to the diode laser.

[15] Keopsys SA, 21, rue Louis de Broglie, 22300 Lannion, France; also Keopsys Inc., Suite 200, 3055 Prosperity Avenue, Fairfax, VA 22031; http://www.keopsys.com

[16] Stratophase Ltd, Unit A7, The Premier Centre, Premier Way, Romsey, Hampshire S051 9DG, United Kingdom; http://www.stratophase.com

[17] P. A. Champert, S. V. Popov, and J. R. Taylor, Electron. Lett. 37, 1127 (2001).

[18] A. Liu, M. A. Norsen, and R. D. Mead, Opt. Lett. 30, 67 (2005). 